\title{
Correction for accurate instantaneous emission measurements of passenger cars
}

\author{
T. Le Anh, S. Hausberger \& M. Zallinger \\ Institute for Internal Combustion Engines and Thermodynamics, \\ Graz University of Technology, Austria
}

\begin{abstract}
Instantaneous emission models use emission measurements of vehicles or engines in transient test cycles in the form of emission maps (velocity/acceleration or engine torque/engine speed). The advantage of this method is that a complete map can be gained from one test cycle. Since the engine map is available, it is possible to integrate new, unmeasured driving patterns over the model and calculate their emission factors without further measurements. However, instantaneous emission models need a precise time alignment between the emission value and the engine load. Otherwise systematic errors will occur in the resulting emission maps.

Because of analyzer's response time, a variable exhaust gas flow leading to a variable transport time of the exhaust gas to the analyzer, and mixing processes within the exhaust gas lines, it is crucial to correct the measured data before using them in the instantaneous emission models.

This paper presents a CFD simulation of emission transport from engine-out to the analyzer, whereby impacts which cause emission signal misalignment and smoothing are investigated. A correction method via mathematical functions is given to align analyzer signals to engine-out by compensating the variable transport time and to reconstruct those signals closer to their original formation by compensating the signal smoothing. The variable time shift of undiluted exhaust gas has a significant role especially for systems with a long exhaust pipe, and in the case of an engine running with high variable speed and load.

This correction method was successfully validated within the ARTEMIS (Assessment and Reliability of transport Emission Models and Inventory Systems, http://www.trl.co.uk/ARTEMIS) project by means of PHEM, an instantaneous emission model based on engine emission maps created and applied recently at TU-Graz.

Keywords: instantaneous emissions, emission signal correction, instantaneous emission model, engine map, emission movement modeling.
\end{abstract}




\section{Introduction}

Many current instantaneous emission models are based on engine maps gained from instantaneous emission measurements. The mapping of emission is performed by statically relating the emission signals to causative variables such as engine speed and engine load. As a result of this static approach, the emission values can be correlated to the correct engine operating state only if they are at the correct location on the time scale. However, beside malfunctions of the exhaust sampling systems and potential operating mistakes, there are several potential systematic problems associated with the instantaneous emission measurement. The emissions recorded by the CVS (Constant Volume Sampling) analyzers are delayed and smoothed compared to the emission events at the location of formation due to:

- The transport of the exhaust gas to the analyzer,

- The mixing of the exhaust gases especially in the silencer and the CVS tunnel,

- The response time of the analyzers.

The transport time of the exhaust gas to the analyzer is determined by the instantaneous flow velocity in the exhaust sampling system and the analyzer response. According to engine operating conditions such as engine speed and load, the undiluted exhaust gas volume flow is variable, which results in variable velocity of the undiluted exhaust gas.

All together, the variable transport time and the analyzer response time can shift the emission signal from approximately 6.5 to 25 seconds (depending on the engine capacity, the vehicle exhaust pipe, the CVS system, the analyzer used and certainly the engine speed and load). Moreover, mixing processes during the gas transport and the analyzer response behavior add a smoothing effect on the signals of the exhaust gas concentration levels.

Although this issue is well known in the area of vehicle emissions testing and modeling, very little research on the aspects of correcting these misalignments can be found in published literature.

Research on instantaneous emission measurements on engine test benches for heavy duty engines was early addressed by Jost et al. [5]. Recently, it was mentioned by Hausberger [3], wherein an empirical correction method was used to align the instantaneous emission measurements at engine-out by simple constant time-shift and variable time-shift by means of an Arctan function, which helps also to reconstruct the emission level smoothed by any effect happening within the transport time of exhaust gas.

The influence of instantaneous speed and acceleration on hot passenger car emissions and fuel consumption was mentioned by Joumard et al. [6] and André and Pronello [1], of which a model was given in the form of a two-dimensional function with the variables speed and speed times acceleration. However, the alignment of the instantaneous emissions with those variables was not found and the model was limited only for urban conditions.

For accurate instantaneous emission measurement, the describing and compensating gas transport dynamics process was done quite in detail by 
Weilenmann et al. [8], then developed by Ajtay and Weilenmann [2]. In their research, the gas transport dynamics process was compensated by applying the mathematical functions such as first and second order time-delay functions with a variety of constant and variable factors, which take into account the engine speed and engine load in their steady state manner. This method is called EMPA correction method [7].

This paper presents a simple and flexible method for correcting the instantaneous emission signals measured on two roller test benches available at TU-Graz. This method is based on:

- Explaining the change of the emission value from their location of formation to the analyzer signal by formulas based on experimental analysis and simulation of the exhaust gas sampling system,

- Inverting these formulas to gain equations which transform the CVS analyzer signal into the engine-out emission value.

This method was validated and finalized in the ARTEMIS project, WP300 task 3156 [7] and task 332 [9]. It has been proved that the correction method has improved significantly the quality of instantaneous emission signals. The improvement can be seen in the reasonable engine maps and on the results of the instantaneous emission models.

\section{Model of the CVS system}

The exhaust sampling system from engine-out to the analyzer's signal is divided into reasonable parts, so that for each part the transport mechanisms can be simulated and clarified (Figure 1).

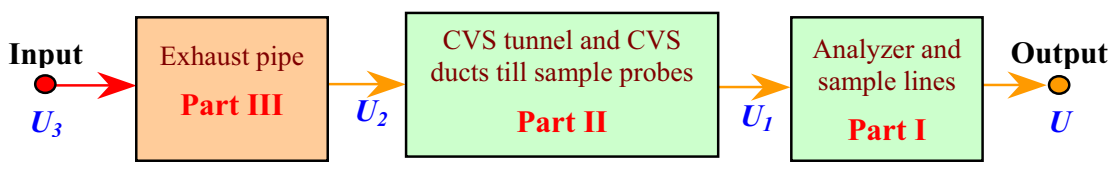

Figure 1: Simplified model of the exhaust gas transport.

In Figure 1, $U, U_{1}, U_{2}, U_{3}$ are emission concentrations at analyzer output, sampling probe, tailpipe/mixing-T and engine-out, respectively. The term $U_{2}$ denotes either undiluted exhaust gas signal at the tailpipe when it belongs to Part III (undiluted side), or diluted gas signal at mixing-T when it is an input signal for Part II (diluted side). These two concepts differ from each other by a multiplier DF (dilution factor).

For each part, the transport of exhaust gases covers two processes: "simple transport" and "dynamics". The first term implies the movement with a constant volume flow that produces a time-delay of the recorded signal, whereas the "dynamics" implies all other impacts, which influence the flow characteristics such as mixing, turbulence, reaction, condensation, etc. that determines the smoothing of the measured signal. 


\section{Simulation of the exhaust gas flow by means of CFD}

This chapter gives an overview of the exhaust gas transport and all effects on emission measurement such as pipe geometry, and velocity, mixing and turbulence of the flow. In particular, the transport of one emission component was investigated. For this purpose, a 3D CFD model was created in FLUENT software (Figure 2) [7].

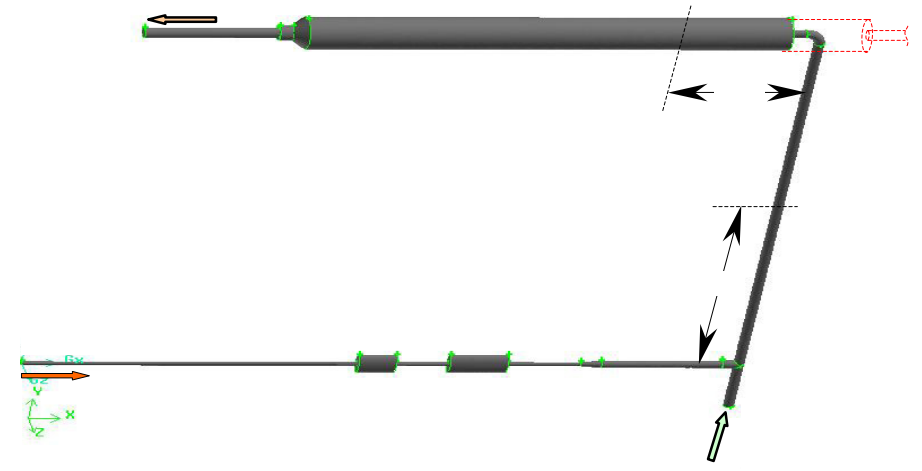

Figure 2: Model of the exhaust gas transport.

The model is a transient segregated, implicit one, which consists of $215^{\prime} 251$ cells and uses standard k- $\varepsilon$ turbulence closure. $\mathrm{CH}_{4}$ species denoting $\mathrm{HC}$ emission is used as a second species together with air.

Figure 3 gives an overview of the effects of turbulent and mixing processes in the entire exhaust pipe of a passenger car. Investigating the movement of one emission peak through the exhaust pipe via 4 time steps, T1,T2, T3 and T4 $(H C(T 1), \ldots, H C(T 4)$, correspondingly), the influence of the pipe geometry and the turbulence (denoting by turbulent intensity-Tur (T1) curve) of the flow on the emission movement and the emission peak flattening is clearly shown. The strong mixing inside the muffler and the robust turbulence right after the muffler-out cause significant smoothing of the emission peak and extend the time-delay of the emission signal.

In Figure 4, the $\mathrm{HC}$ fraction was recorded by the simulation at different cross sections of the model. As the velocity of the flow at Inlet 1 is high, the first three peaks need about 1 second to move from Inlet 1 to $x o z$ cross section, while it is 3 seconds for the last HC peak, when the Inlet 1 velocity is low. However, each of the four $\mathrm{HC}$ fraction peaks needs equally 2 seconds to transport from $x o z$ cross section to model Outlet due to constant volume flow in the mixing pipe and the CVS tunnel.

The simulation also shows that, even though there is a big difference in the flow distribution in the exhaust pipe due to the difference of velocity at model Inlet 1 , it is really similar in the mixing pipe and the CVS tunnel. The similarity of the velocity distribution in the mixing pipe and the CVS tunnel therefore leads 
to a potentially constant time-delay of the diluted gas moving in those parts of the exhaust sampling system [7].

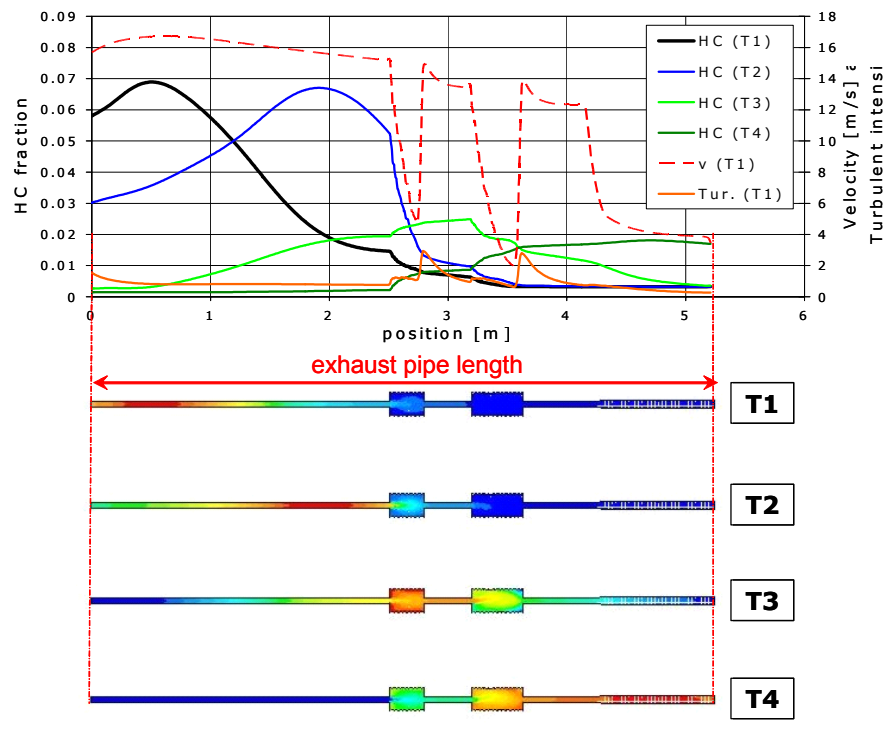

Figure 3: CFD-Simulation results on the movement of a HC-emission peak through the exhaust pipe of a car.

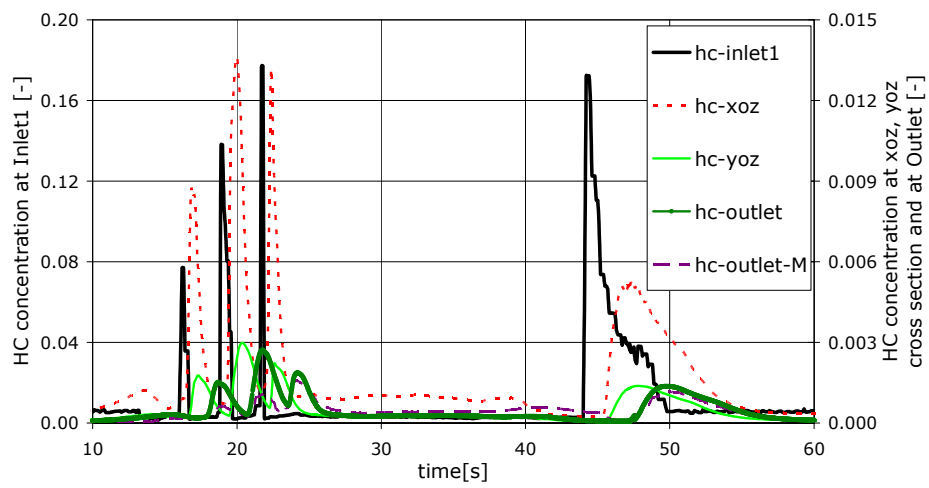

Figure 4: $\quad$ Measured HC fraction signal at model Inlet1 (hc-inlet1), Outlet (hc-outlet-M), and simulated $\mathrm{HC}$ fraction signals at cross sections xoz (hc-xoz) and yoz (hc-yoz), and at model Outlet (hc-outlet).

Because of the constant volume flow in the mixing pipe and the CVS tunnel, only the smoothing and the constant time-delay have to be considered in those parts. In the exhaust pipe, the time-delay is however variable, depending on the exhaust gas volume flow and the exhaust pipe length. Thus, the instantaneous emissions should be corrected to prevent systematical errors in the time alignment. 


\section{Inversion of the exhaust gas transport model}

The inversion of the exhaust gas transport model is conducted in turn for each of the three parts in Figure 1, which targets to compensate for the signal delay and signal smoothing due to the afore mentioned reasons, including the correction for instantaneous diluted exhaust gas and for instantaneous undiluted exhaust gas signals. A detailed description can be found in [7].

\subsection{Correction for the diluted exhaust gas signals}

According to the simulation results, the instantaneous CVS analyzer signal $(U)$ is corrected to align at sampling probe (the so-called $U_{I}$ signal) by using a combined Arctan-PT2 function; whereby, the analyzer response and sampling gas dynamics in Part $I$ are compensated.

$$
U_{1}(t)=U(t)+A_{1} \sqrt{\left|U^{\prime}(t)\right|} \arctan \left[C_{1} U^{\prime}(t)\right]+B_{1} U^{\prime \prime}(t) .
$$

Here $A_{l}, C_{l}$ and $B_{l}$ are constants.

The delay time element function second order (PT2) is used to compensate for the signal smoothing in Part II.

$$
U_{2}(t)=U_{1}(t)+A_{2} U_{1}^{\prime}(t)+B_{2} U_{1}^{\prime \prime}(t) .
$$

Here $A_{2}$ and $B_{2}$ are constants, $U_{2}$ is the instantaneous emission or fuel consumption signal at mixing-T.

Coefficients $A_{1}, C_{1}, B_{1}, A_{2}$ and $B_{2}$ were initially gained by applying functions (1) and (2) to correct the analyzer signals gained from calibration gases, which were inserted at the mixing position of exhaust gas and dilution air. Those coefficients were then modified to apply for standard measurement data (instantaneous emission data recorded by the CVS analyzers while the cars were driven following standard driving cycles on a roller test bench) to have final values [7].

\subsection{Variable time shift for undiluted exhaust gas signals}

The variable time-delay in the exhaust pipe is determined by a power function of the exhaust gas volume flow as follows:

$$
\Delta t=K\left(V_{e x h}\right)^{M} .
$$

Here $\Delta t$ is the time step, of which the analyzer signal has to be shifted; $K$ and $M$ are constants depending on the total exhaust pipe length, which is counted from engine-out to tailpipe, right before the mixing position of exhaust gas and dilution air.

Function (3) is a simple and efficient solution since the exhaust gas volume flow $\left(V_{e x h}\right)$ can be gained from direct measurement or from dilution factor (DF) calculated via measured instantaneous emissions. Because a direct measurement of the exhaust gas volume flow is not available for most standard emission tests for cars around Europe, the second way is of interest. Using the measured instantaneous emissions to calculate the exhaust gas volume flow from the DF is 
accurate only if the engine operates close to stoichiometric combustion $(\lambda=1)$. For such engines a correction can be applied without measuring additional values compared to the usual test bed operation. However, for diesel cars, the variability in transport time of the undiluted exhaust gas, which can be determined by adding lambda or $\mathrm{CO}_{2}$ sensors in the undiluted exhaust gas [7], is much smaller than for gasoline cars. The variable time-delay can be replaced satisfactorily by constant time shift. Figure 5 depicts a correlation between time-delay $(\Delta t)$ and the exhaust gas volume flow of a CFV-CVS (Critical Flow Venturi-Constant Volume Sampling) system with the total exhaust pipe length of approximately 15 meters.

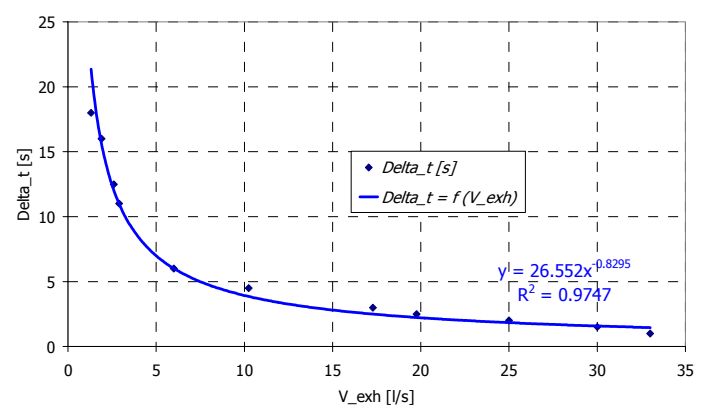

Figure 5: Time-delay on the total exhaust pipe of the CFV-CVS system as a function of the exhaust gas volume flow.

\subsection{The complete inversion model}

The complete inversion model for gasoline passenger cars includes the following four steps:

1. Constant time shift to align roughly the analyzer signal to the tailpipe.

2. Application of the combined Arctan-PT2 function (1) to compensate for the analyzer dynamics and the diluted exhaust gas dynamics in Part $I$.

3. Application of the PT2 function (2) to compensate for the diluted exhaust dynamics in Part II.

4. Application of the variable time shift (3) to align the analyzer signal to engine-out.

Since the change of the exhaust volume flow of diesel cars is quite small, step 4 can be omitted, and as for step 1, the analyzer signal is aligned to engine-out by a constant time shift.

\section{Validation of the inversion model}

In order to validate the correction method, an instantaneous emission model PHEM (Passenger car and Heavy duty vehicle Emission Model) is used. A corrected fuel consumption signal at catalyst-out of a EURO 2 car is firstly compared with the original measured one and with the engine power curve. Then engine maps generated by PHEM are analyzed. 


\subsection{Instantaneous emission model PHEM}

The model PHEM was developed at the TU-Graz in the year 2000 and has been continuously improved. In order to prepare a more reliable basis for PHEM to apply accurately for modern passenger cars, the instantaneous emissions are corrected to eliminate signal misalignment and to compensate for signal smoothing.

With a given driving cycle and road gradient, the effective engine power is calculated in $1 \mathrm{~Hz}$ frequency from the driving resistances and losses in the transmission system. The actual engine speed is simulated by the transmission ratios and a driver's gear shift model. The emissions are then interpolated from engine maps (Figure 6).

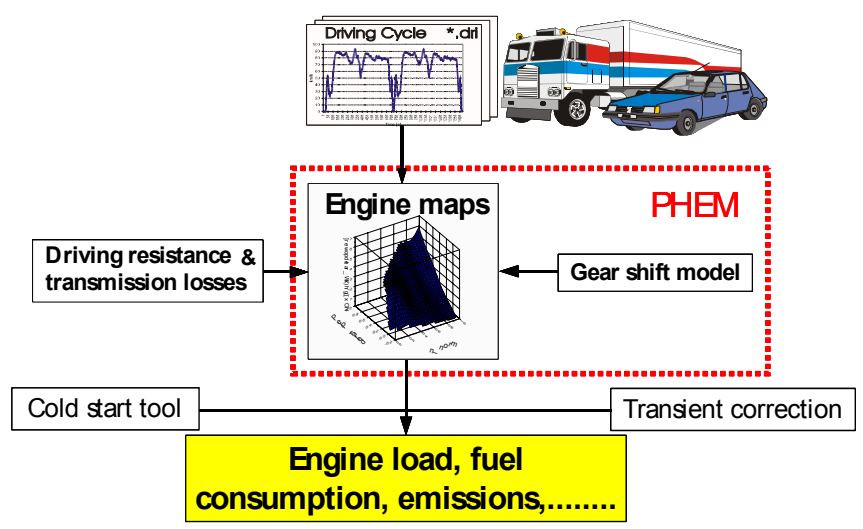

Figure 6: Schematic picture of the model PHEM.

The model is capable of modeling passenger cars and heavy duty vehicles. Data for the average vehicle fleet as well as for single vehicle are available in [9] and [10].

\subsection{Validation of the correction method}

The correction significantly improved the quality of the instantaneous emission signals as well as the instantaneous emission model denoted by the correct engine maps.

Figure 7 shows an example of the correction method applied for a data set measured by the CFV-CVS system.

In the presented time period in Figure 7, the correction has eliminated the misalignment of FC signal from 8 to 18.5 seconds. Consequently, the corrected FC signal ( $F C$, final correction) aligns well with the engine power signal $\left(\mathrm{Pe} / \mathrm{P}_{\text {rated }}\right.$. Moreover, the correction has also moderately compensated for the smoothing process depicted by the emission peaks.

Engine maps were built up by PHEM based on fully corrected signals (applied four steps correction method for gasoline cars or three steps correction 
method for diesel cars) and on signals applying only constant time shift, for comparison (Figure 8, left and right, respectively).

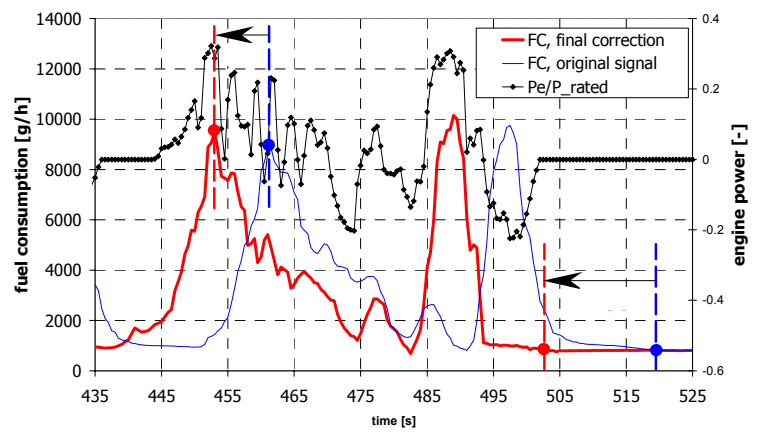

Figure 7: Correction for fuel consumption (FC) signal calculated via Cbalance method of a EURO 2 gasoline car.

The engine map gained from the fully corrected FC signal is much more reasonable than the map gained from the uncorrected measurement signal, because the uncorrected signal leads to FC values of less than 200 [g/kWh] in areas of low engine speeds and high engine loads (Figure 8, right), a value not achieved so far from gasoline engines in serial production. This low specific fuel consumption results from systematic misalignments between engine power and fuel consumption measurement [7].

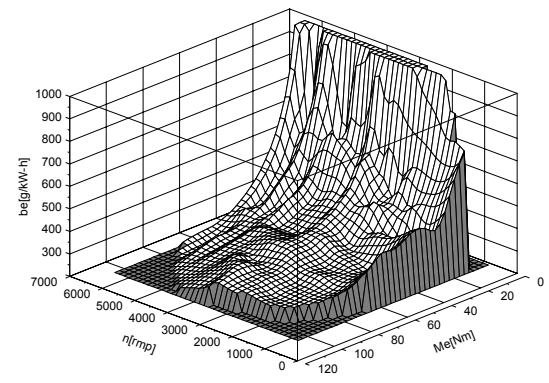

(Application of full correction method)

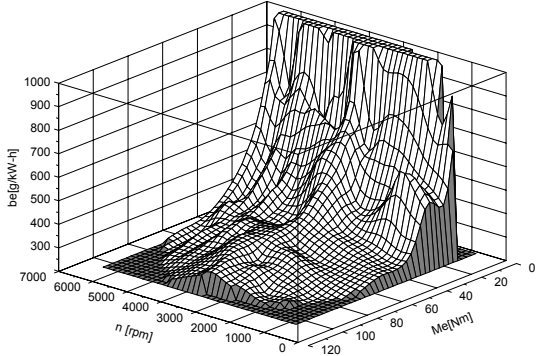

(Application of only constant time shift)

Figure 8: 3D fuel consumption map of a EURO 3 gasoline car with and without full correction of the instantaneous measurement signal.

\section{Conclusion}

When instantaneous emission measurements in transient cycles are used to set up emission maps for instantaneous emission models, systematical errors can occur due to the different response time characteristics of the measuring chain for exhaust gas on one hand and engine speed and engine load (or vehicle speed and acceleration) on the other hand. 
While the response time for the exhaust gas is variable and the signal is smoothed, the signals for engine speed and engine load have nearly no response time and are not smoothed. Thus the time alignment of the emission signals with engine power and engine speed events would be frequently wrong and emission levels are not allocated correctly to the engine loads in the maps, if no correction method is applied.

An inversion model is presented to rebuild the original "engine-out" emission value from the measured emission curve of the analyzers located after the CVStunnel. This is done in the model by the inversion of the transmission mechanism of emission signals from engine-out to analyzer response. In other words, the inversion model is created to compensate for all effects on emission transport such as transport time, mixing, turbulence and convolution.

The correction method developed clearly improved the quality of the engine maps, together with other recent improvements [9], the emission factors generated by the model PHEM agree well with the measured ones.

\section{References}

[1] André, M., Pronello, C., 1996: Speed and acceleration impact on pollution emissions; SAE paper, 961113. 1996.

[2] Ajtay, D., Weilenmann, M., 2004: Compensation of the exhaust gas transport dynamics for accurate instantaneous emission measurements; EMPA, 2004.

[3] Hausberger S., 2003: Simulation of Real World Vehicle Exhaust Emissions; VKM-THD Mitteilungen; Heft/Volume 82; Verlag der Technischen Universität Graz; ISBN 3-901351-74-4; Graz 2003

[4] FLUENT user guide, chapters 6, 10, 12, 13.

[5] Jost, P., Hanssel, D., Weber, F-J, Sonnborn, K-S, 1992: Emission and fuel consumption modeling based on continuous measurements; Deliverable No. 7 of the Drive Project V 1053.TÜV Rheinland, Cologne, FRG, 42p, 1992.

[6] Joumard, R., Jost, P., Hickman, J., 1995: Influence of instantaneous speed and acceleration on hot passenger car emissions and fuel consumption; SAE paper 950928. 1995.

[7] Le Anh, T., Hausberger, S., Ajtay, D., Weilenmann, M., 2005: Response times in instantaneous emission measurement; ARTEMIS WP300-Task 3156, final report, Graz 02/2005.

[8] Weilemann, M., Soltic, P., Ajtay, D., 2002: Describing and compensating gas transport dynamics for accurate instantaneous emission measurement; International Symposium "Transport and air pollution", Graz, Austria, Vol. 1 p155-162, 2002.

[9] Zallinger M., Hausberger S., Ajtay, D., Weilemenn, M., 2005: Instantaneous emission modeling applications; ARTEMIS, WP300 - Task 332, final report, Graz 02/2005.

[10] Rexei, M. Hauberger, S., et al., 2005: Heavy Duty vehicle Emissions; ARTEMIS, WP400, final report, Graz 02/2005. 